\title{
CONSUMO EXTRA DE OXIGÊNIO APÓS EXERCíCIOS AQUÁTICOS, EM CICLOERGOMETRO E DE RESISTÊNCIA
}

\author{
Renan Marcondes Porto, Jair Rodrigues Garcia Junior \\ Curso de Educação Física da UNOESTE, Presidente Prudente, SP.
}

\section{RESUMO}

O consumo extra de oxigênio pós-exercício (EPOC) representa um gasto energético adicional àquele que ocorre durante o exercício, por isso, tem sido medido durante várias horas após a prática de diferentes exercícios. O objetivo deste estudo foi revisar, descrever, comparar e discutir o EPOC resultante da prática de exercícios aquáticos, em cicloergômetro e de resistência. Foi realizado levantamento dos artigos nas bases de indexação, priorizando aqueles publicados a partir de 2006. Foi verificado que há diversos fatores que influenciam o EPOC, principalmente a intensidade e duração do exercício. Exercícios com intensidade de, pelo menos $70 \%$ do $\mathrm{VO}_{2}$ máx. e duração de 60 min provocam maior EPOC. O tempo do EPOC pode se prolongar de $2 \mathrm{~h}$ até $48 \mathrm{~h}$ e o valor do consumo de oxigênio pode chegar a $23 \mathrm{~L}$ ou 114 Kcal, após exercício resistido. No cicloergômetro com intensidade de $60 \%$ do $\mathrm{VO}_{2}$ máx. e duração de 20 min, o EPOC médio nos primeiros 16 min de recuperação é de 2,9 L/min. Em vista da intensidade e duração apresentarem grande variação nos exercícios estudados, as comparações ficam dificultadas, porém, os resultados indicam que o EPOC é maior em exercícios aquáticos, seguidos pelo cicloergômetro e pelos exercícios resistidos. Assim, pode-se concluir que o EPOC sofre influência de vários fatores, principalmente do tipo de exercício, intensidade e duração. Esses dois últimos fatores podem fazer com que o EPOC represente um consumo adicional de oxigênio correspondente a 10-20\% daquele que ocorreu durante a sessão de exercício.

Palavras-chave: consumo de oxigênio, exercício, gasto energético, EPOC, treinamento.

\section{EXCESS OF POST-EXERCISE OXYGEN CONSUMPTION AFTER WATER, CYCLE ERGOMETER AND RESISTANCE EXERCISES}

\begin{abstract}
The excess of post-exercise oxygen consumption (EPOC) represents an energy expenditure added to exercise's energy expenditure, so, it was been measured by hours after practice of different exercises. The aim of this study was to review, describe, compair and discuss EPOC resulting of water, cycle ergometer and resistance exercises practice. It was carried out a search by papers on index basis, selecting mainly these ones published after 2006. It was observed several factors that take effects on EPOC, mainly exercise intensity and duration. Exercise intensity on $70 \%$ of $\mathrm{VO}_{2}$ máx. and duration of 60 min induces the higher effects on EPOC. Duration of EPOC can be extend by $2 \mathrm{~h}$ until $48 \mathrm{~h}$ and the oxygen consumption can be reach $23 \mathrm{~L}$ or $114 \mathrm{Kcal}$, after resistance exercise. On cycle ergometer with intensity of $60 \%$ of $\mathrm{VO}_{2}$ máx. and duration of $20 \mathrm{~min}$, average EPOC on first $16 \mathrm{~min}$ of recovery is $2.9 \mathrm{~L} / \mathrm{min}$. As the intensity and duration show large variation on studied exercises, it is difficult the comparision, however, the results indicated that EPOC is higher on water exercises, followed by cycle ergometer and resistance exercises. Thus, it is concluded that EPOC is influenced by several factors, mainly exercise type, intensity and duration. These two last ones can make EPOC represent additional oxygen consumption as $10-20 \%$ to oxygen consuption during exercise.
\end{abstract}

Keywords: oxygen consumption, exercise, expenditure energetic, EPOC, training. 


\section{INTRODUÇÃO}

O gasto energético e o consumo extra de oxigênio pós-exercício (EPOC - excess of postexercise oxygen consuption) vêem ganhando mais espaço e importância, pois há interesse da população em geral e vários estudos sendo realizados, com a descoberta de novos dados. Gaesser e Brooks (1984) foram os primeiros estudiosos a realizar esse tipo de estudo, tendo a preocupação em descobrir o motivo da taxa metabólica permanecer elevada mesmo após o exercício. A maneira que eles encontraram para realizar essa analise foi a medição do consumo de oxigênio e, por isso a taxa metabólica aumentada foi denominada de EPOC. Portanto, o EPOC é o gasto energético aumentado após o exercício, que ocorre em razão dos sistemas fisiológicos buscarem o restabelecimento da homeostase.

Existem vários fatores que podem influenciar o EPOC, como massa muscular envolvida no exercício, intensidade, duração, estado do treinamento, ingestão de alimento (efeito térmico da refeição), qualidade do sono da noite anterior e condições ambientais, entre outros (Imamura et al., 2004). São fatores que devem ser considerados ao analisar e comparar os estudos sobre o EPOC.

Dessa forma, o objetivo deste estudo foi o de revisar, descrever, comparar e discutir o EPOC resultante da prática de exercícios aquáticos, em cicloergômetro e de musculação.

\section{MÉTODOS}

A partir das palavras chave EPOC, consumo extra de oxigênio, consumo de oxigênio, exercício físico, recuperação, gasto energético, pós-exercício, $\mathrm{VO}_{2}$ e atividade física, assim como os termos equivalentes em inglês, foi realizado o levantamento dos artigos nas bases de indexação MedLine, SciELO e Lilacs, priorizando aqueles publicados de 2006 a 2011. As informações foram analisadas, descritas, comparadas e discutidas.

\section{Consumo de oxigênio pós-exercício (EPOC)}

Quando se realiza qualquer tipo de exercício físico, seja uma simples caminhada até o trabalho ou um treinamento programado constituído por exercícios de elevada intensidade, há aumento no consumo de oxigênio em razão do aumento da demanda de vários órgãos e, principalmente dos músculos. Durante o exercício, o consumo aumenta em proporção à intensidade do exercício e, permanece aumentado mesmo após o final do esforço (Figura 1). Essa constatação foi feita inicialmente por Gaesser e Brooks (1984), ao estudarem a dinâmica do consumo de oxigênio após a realização de exercícios aeróbicos e de contraresistência, observando que a taxa metabólica permaneceu elevada em relação aos valores de repouso. O consumo aumentado de oxigênio mesmo sem o esforço muscular é necessário para que o organismo retorne ao seu estado de homeostase e, esse aumento da taxa metabólica foi denominado de excess post-exercise oxygen consumption (EPOC) (Gaesser e Brooks, 1984) ou de débito de oxigênio (Foureax et al., 2006).

O consumo de oxigênio permanece elevado porque o esforço físico provoca a perda da homeostase, alterações da temperatura, da concentração de íons e de substratos. Esses desequilíbrios exigem esforços maiores dos sistemas fisiológicos (cardiovasculares, respiratórios, endócrino e outros), fazendo com que consumam mais oxigênio. Em exercício de intensidade maxima, o desequilíbrio é de tal magnitude que não há condições de estabelecer o estado estável, por isso, ocorre fadiga precoce (Figura 2). Lecheminant et al. (2008) observaram que esse aumento do consumo de oxigênio provocado pela sessão de exercício é transitório e não se mantém por mais de 24 horas. 


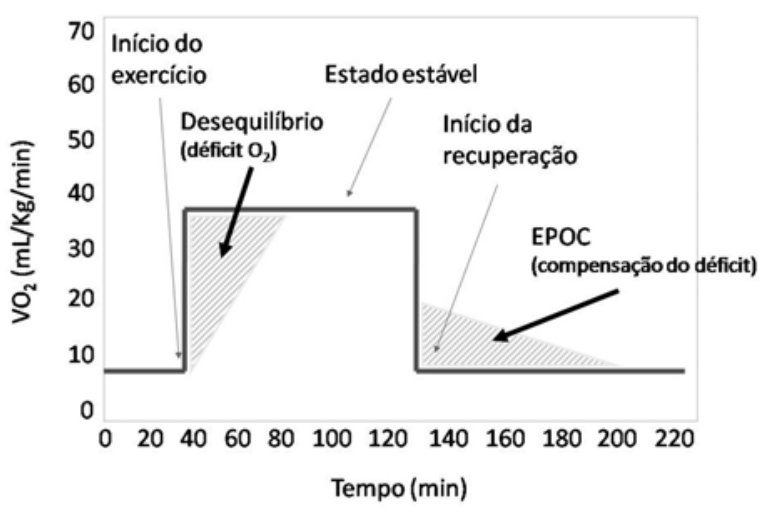

Figura 1. Gráfico demonstrativo do consumo de oxigênio em repouso, durante o exercício e na recuperação. $\mathrm{Na}$ prática de exercício de intensidade moderada, no início há um desequilíbrio e ocorre um déficit de oxigênio até que seja estabelecida a condição de estado estável. O déficit é compensado a partir do final do exercício com o consumo extra de oxigênio pós-exercício (EPOC).

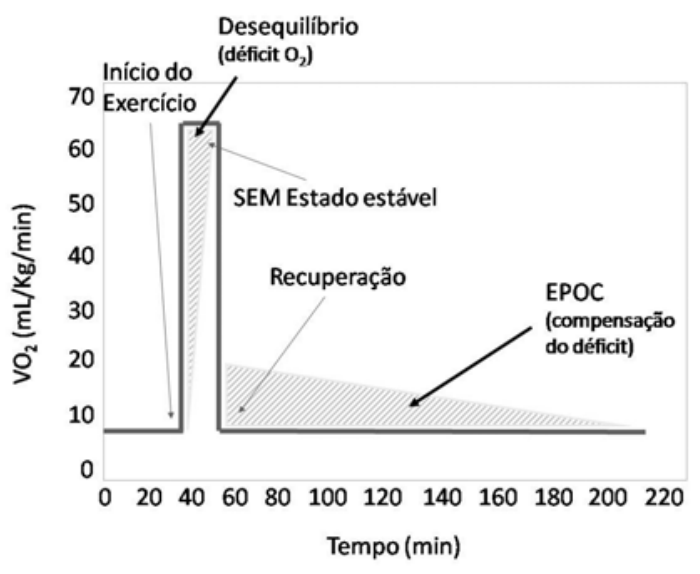

Figura 2. Gráfico demonstrativo do consumo de oxigênio em repouso, durante o exercício e na recuperação. $\mathrm{Na}$ prática de exercício de intensidade máxima, no início já há desequilíbrio de elevada magnitude, ocorre um déficit de oxigênio e não há condições de estabelecer a condição de estado estável, ocorrendo a fadiga. O déficit é compensado a partir do final do exercício com o consumo extra de oxigênio pósexercício (EPOC).

O consumo extra de oxigênio após o exercício (EPOC) pode ser medido de duas maneiras: por calorimetria indireta, ou seja, a utilização de uma máscara ou bucal ligados a um aparelho que coleta e analisa o oxigênio que está sendo consumido e, a calorimetria direta, com a utilização de uma câmara onde o indivíduo permanece isolado e tem o calor de seu corpo medido para o cálculo do consumo de oxigênio e gasto energético (McARDLE et al., 2008).

O EPOC pode ser dividido em três componentes: o componente rápido com duração de 10 seg a alguns minutos; o componente lento, que pode durar várias horas e, por fim, o componente ultralento, que refere-se à taxa metabólica elevada que pode perdurar por várias horas (Figura 3) (BØSHEIMM et al., 1998; DOLEZAL et al., 2000). A duração dos três componentes depende do grau do distúrbio da homeostase causado pelo esforço, podendo ser observados os efeitos de vários hormônios, como o cortisol, adrenalina, adrenocorticotrófico (ACTH), hormônios da tireóide e hormônio do crescimento (GH) (THORTON; POTTEIGER, 2002).

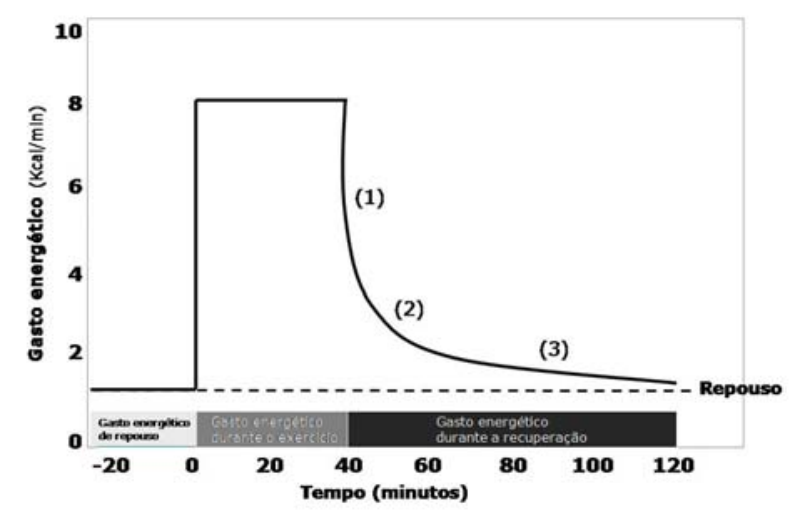

Figura 3. Gráfico demonstrativo do gasto energético durante o repouso, exercício e a recuperação com os componentes (1) rápido, (2) lento e (3) ultralento do consumo extra de oxigênio pós-exercício (EPOC).

Pode ser observado em vários testes realizados sobre a duração do EPOC, que os maiores valores do consumo de oxigênio após o exercício são encontrados nos minutos iniciais da fase de recuperação, isto é, a fase rápida de diminuição da taxa metabólica. Durante a fase lenta os valores são muito menos pronunciados, embora nessa fase ainda existam processos que proporcionam a restauração da homeostase, 
porém, requerem menor captação de oxigênio do que os processos que ocorrem na fase rápida (THORTON; POTTEIGER, 2002).

Mesmo sendo estudado há muitos anos, pode ser observado que há ainda muito que se pesquisar sobre o EPOC, principalmente o tempo em que permanece elevado, a magnitude e duração do EPOC. O que se pode concluir inicialmente é que o EPOC representa um componente de gasto de energia em qualquer tipo de exercício físico, independentemente da duração e intensidade, que são as variáveis mais determinantes da magnitude e duração do EPOC.

\section{Fatores determinantes do EPOC}

O motivo pelo qual surgiram controvérsias sobre o tempo de duração do EPOC está relacionado aos fatores que podem influenciar a realização dos exercícios. Imamura et al. (2004) mencionam alguns fatores importantes que podem determinar a diferença da duração do EPOC: massa muscular envolvida no exercício, intensidade e duração, estado do treinamento, ingestão de alimento (efeito térmico da refeição), qualidade do sono da noite anterior, condições ambientais, familiaridade do sujeito com $\mathrm{o}$ protocolo, variações na temperatura e nas concentrações de catecolaminas, custo metabólico da remoção do lactato, utilização de substrato e fase do ciclo menstrual das mulheres, ansiedade pré-exercício e ritmo circadiano (Figura 4). Meirelles e Gomes (2004) afirmam ser a intensidade do exercício o fator mais determinante na magnitude do consumo excessivo de oxigênio após o exercício.

Além dos estudos de Thorton e Potteiger (2002), Meirelles e Gomes (2004) têm demostrado que exercícios de alta intensidade produzem maior elevação no EPOC do que exercícios de menor intensidade, devido ao fato dos primeiros causarem maior estresse metabólico, sendo necessário então, maior gasto de energia para retornar à homeostase. Essa idéia é comprovada também por Imamura et al. (2004), que demonstraram ser o EPOC relativamente curto após exercícios de intensidade e duração moderada, ou seja, menor que $70 \%$ do $\mathrm{VO}_{2}$ máx. e de menos que $60 \mathrm{~min}$. Por outro lado, quando o exercício é mais prolongado (acima de uma hora) e mais aeróbio, pode causar um EPOC mais prolongado. Nesse caso, os sistemas fisiológicos estabelecem uma condição de estado estável após os primeiro minutos de esforço e adaptações hemodinâmicas e metabólicas agudas, porém, o tempo inicial para adaptação provoca uma "dívida de oxigênio" que só poderá ser paga após o final do exercício (Figura 1).

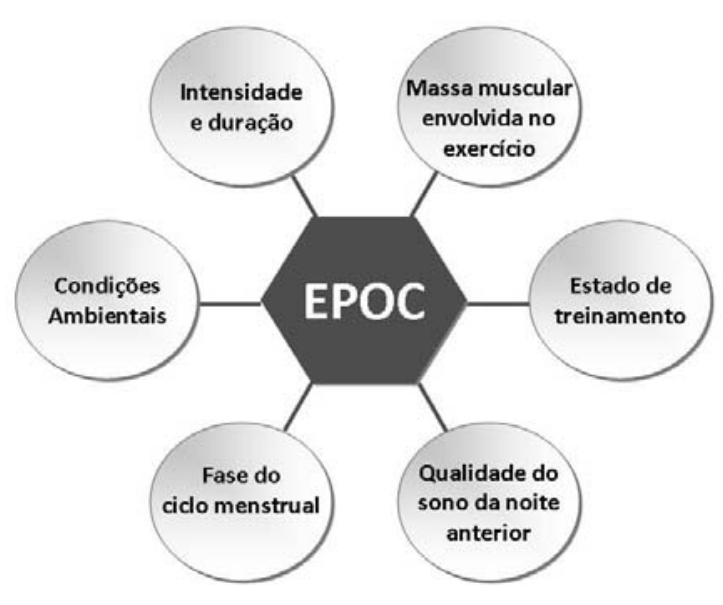

Figura 4. Fatores que influenciam o consumo extra de oxigênio pós-exercício (EPOC).

Então, quanto mais prolongado e mais intenso for o exercício, maior desequilíbrio provocado, maior a divida de oxigênio e por mais tempo seu consumo permanece elevado durante a recuperação. Por isso, exercícios praticados ao longo de duas horas ou mais, em intensidade entre os limites de moderada e alta, fazem com que o gasto energético e, obviamente, o consumo de oxigênio voltem ao valor de repouso apenas depois de 20 horas ou mais (LeCHEMINANT et 
al., 2008). O EPOC prolongado pode ser considerado uma vantagem para indivíduos que buscam aumentar seu gasto energético, porém para que o indivíduo realize uma sessão de exercício prolongada com intensidade limite entre aerobiose e anaerobiose deve estar muito bem condicionado físicamente (Matsuura et al., 2006).

Outro fator que pode influenciar o consumo excessivo de oxigênio após o exercício é o nível de treinamento (Lecheminant et al., 2008). O EPOC passou a ser estudado também na prática de exercícios resistidos, nos quais a intensidade pode ser determinada pela carga utilizada e também pelo intervalo entre as séries, além de haver o fator duração. Estudo realizado por Schuenke et al. (2002) demonstrou que o EPOC permaneceu elevado para além de 16 horas após a realização de um exercício resistido de apenas 38 seg. Porém, é necessário considerar a impossibilidade de manter 0 indivíduo em repouso e emocionalmente estável ao longo de períodos prolongados, por isso, podem haver pequenos aumentos do consumo de oxigênio ao longo do dia ou de um período avaliado, como consequência das atividades cotidianas, alterações emocionais e do próprio ritmo circadiano. Estes são aspectos que não são controlados na maioria dos estudos.

\section{Exercícios aquáticos e EPOC}

Os exercícios aquáticos como hidroginástica e natação, há muito tempo são indicados pelos profissionais de saúde como forma de melhorar a saúde e também como forma de reabilitação, por não haver muito impacto com o solo, mas sim certa dificuldade em realizar movimentos no meio líquido em razão da resistência da água, sendo necessária mais força e levando ao fortalecimento músuscular. Atividades como hidroginástica ou qualquer exercício em meio líquido realizados na posição vertical proporcionam diversos benefícios à saúde, ao mesmo tempo em que provocam número muito menor de lesões osteomioarticulares (BAUM, 2000; KRUEL, 2000). Os componentes da aptidão física força, flexibilidade, composição corporal e condicionamento cardiorrespiratório são bastante exigidos nas atividades aquáticas (MULLER, 2002; TAKESHIMA et al., 2002; BARELLA et al., 2004; ALVES et al., 2004). Como a população de idosos é a que mais tende a apresentar diminuição destes componentes, justamente quando também apresentam limitações de movimentos e dores nas articulações, a hidroginástica apresenta-se como ótima opção, desde que os exercícios sejam prescritos adequadamente para tais objetivos.

Somado aos benefícios está o fato de que os exercícios realizados na água provocam comportamentos de freqüência cardíaca e pressão arterial mais estáveis e relativamente mais mais baixos em comparação aos exercícios terrestres (KRUEL, 2000; MIYOSHI et al., 2004). Pinto et al. (2006) realizaram um estudo com dois exercícios de hidroginástica executados com e sem o uso de diferentes equipamentos resistivos nos membros inferiores, avaliando as respostas cardiorrespiratórias. Os resultados demonstraram aumento significativo no consumo de oxigênio com o uso de diferentes equipamentos resistivos para um dos exercícios analisados. A frequência cardíaca também foi significativamente mais elevada com o uso dos equipamentos resistivos para os dois exercícios, comparando com o exercício realizado sem equipamentos. Uma ressalva deste estudo foi a falta de análise das respostas cardiorrespiratórias à utilização de equipamentos nos membros superiores, comparando aos membros inferiores, assim como não foi verificada a sensação subjetiva ao esforço.

No sentido de quantificar o consumo de oxigênio e gasto energético na água, McComb et 
al. (2004) propuseram um método para medir o consumo de oxigênio por meio de um teste de corrida na água. Para validação, os critérios propostos foram verificados e comparados com resultados de testes de esforço máximo que também utilizam a medida direta do consumo de oxigênio.

Na modalidade natação há dificuldades enormes para a medição do consumo de oxigênio e, mesmo com os grandes avanços da tecnologia nessa área, ainda é extremamente difícil à obtenção de uma estimativa válida de parâmetros cardiorrespiratórios. No entanto, se acredita que é de fundamental importância o conhecimento dessas informações, pois a técnica empregada durante o nado pode modificar consideravelmente o gasto de energia no deslocamento do individuo no meio líquido. Por isso, vários pesquisadores começaram a criar maneiras diferentes de medição do consumo de oxigênio em meio líquido. Toussaint et al. (1987) estiveram entre os primeiros a desenvolver um método de medir o consumo de oxigênio por meio de um snorquel modificado e específico para avaliação em natação. Esse método de coleta por meio de um snorquel sofreu modificações adicionais e continua sendo utilizado, inclusive com adaptações para utilização com o equipamento analisador de gases $K 4$ na natação (KESKINEN et al., 2003).

Pode-se observar, então que a dificuldade de medir o consumo de oxigênio em atividades aquáticas é muito grande, tanto na hidroginástica quanto na natação e que os materiais utilizados para tal objetivo ainda estão em evolução. Por esses motivos existem poucos estudos sobre o consumo de oxigênio durante e após o exercício.

\section{Exercícios de resistência e EPOC}

O consumo de oxigênio após o exercício em atividades nas academias inclui não apenas exercícios de resistência, mas também exercícios em esteira e bicicleta ergométrica. O EPOC pode ser útil no planejamento de um programa para atingir determinados objetivos, principalmente a perda do excesso de peso e melhora da saúde. De acordo com Foureaux et al. (2006) exercícios de resistência, como musculação com cargas elevadas, além de proporcionar maior gasto energético por unidade de tempo, também causam maior débito de oxigênio e maior consumo extra de oxigênio após o exercício. Schuenke et al. (2002) mencionam que, durante uma única sessão de exercício de resistência, já foi observado o dispêndio de $864 \mathrm{Kcal}$, quando foram somados 0 valor do gasto energético durante o exercício e o valor do EPOC. Quando são considerados apenas os valores do EPOC, pode haver variação do gasto energético de 6 Kcal a $114 \mathrm{Kcal}$, assim como a duração do EPOC pode variar de $14 \mathrm{~min}$ a $48 \mathrm{~h}$ (DOLEZAL et al., 2000; THORNTON; POTTEIGER, 2002; SCHUENKE et al., 2002). Contudo, é necessário considerar que, na prática de exercícios de resistência, há muitas variáveis incluídas em uma única sessão de exercício.

Em exercícios de resistência, a intensidade é uma das variáveis de maior influência no EPOC, pois o recrutamento de unidades motoras musculares é diretamente proporcional a ela, havendo maior produção de lactato e, consequentemente, maior energia é necessária para sua remoção após o exercício, assim como para o processo de termorregulação que é mantido mesmo ao final do exercício.

Kenny et al. (2003) realizaram estudo sobre alteração da temperatura muscular e central provocada por um exercício de resistência. Após a realização de um exercício de extensão isotônica do joelho com duração de 15 min foi observada elevação na temperatura do músculo vasto lateral de $0,55^{\circ} \mathrm{C}$ a $3,20^{\circ} \mathrm{C}$, a qual se manteve por ate $60 \mathrm{~min}$. De acordo com 
alterações na temperatura é possível inferir a EPOC, já que a produção de calor está diretamente relacionada com o metabolismo energético e consumo de oxigênio. Além disso, a termorregulação posterior ao final do exercício continua impondo maior demanda às glândulas $e$ órgãos, contribuindo para o EPOC.

Thornton e Potteiger (2002) observaram que $O$ EPOC parece ser influenciado pela intensidade, principalmente no componente rápido, quando relacionaram a maior concentração plasmática de lactato em um grupo que realizou exercício de resistência intenso. $O$ EPOC foi medido durante $2 \mathrm{~h}$, sendo significativamente mais elevado após a sessão mais intensa $\left(2,2 \mathrm{~L} \mathrm{O}_{2}\right.$, intensidade de $85 \%$ de 8 RM) em comparação com a menos intensa (1,1 L $\mathrm{O}_{2}$, intensidade de $45 \%$ de $\left.8 \mathrm{RM}\right)$.

Outro estudo de avaliação da EPOC após exercício de resistência foi realizado com uma sessão constituída de três exercícios com quatro séries de 10 RM. Foi observado que o consumo de oxigênio permaneceu elevado por até $38 \mathrm{~h}$ e que, nas $48 \mathrm{~h}$ após a sessão de exercício de resistência, o consumo de oxigênio foi, em média, 0,66 $\mathrm{mL} \mathrm{O} \cdot \mathrm{kg}^{-1} \cdot \mathrm{min}^{-1}$ superior ao consumo de repouso, equivalente a um aumento de $20 \%$ no metabolismo (SCHUENKE et al., 2002). Dessa forma, fica bem estabelecido que a intensidade é de grande influência na magnitude e duração do EPOC, podendo se estender até $48 \mathrm{~h}$ após o final do exercício e representar aumento de até 20\% da taxa metabólica de repouso.

O intervalo de recuperação em qualquer tipo de exercício físico intermitente é inversamente proporcional à intensidade do mesmo, ou seja, um período mais curto de recuperação significa aumento do estresse induzido pelo exercício de forma similar a um aumento de carga (THORNTON; POTTEIGER, 2002). Por isso, o intervalo tem influência no EPOC, como demonstrado num estudo Haltom et al. (1999) que mediu o gasto energético durante uma hora após o termino da sessão de exercício de resistência. Após a sessão com intervalo considerado longo, o gasto energético no período de recuperação foi de $37 \mathrm{Kcal}$, enquanto na sessão com intervalo curto, o gasto energético foi de 52 Kcal. Dessa forma, a mudança do intervalo entre séries pode ser manipulado para provocar diferentes adaptações fisiológicas e metabólicas, além de aumentar também a magnitude e duração do EPOC.

A velocidade de execução dos exercícios é outra variável que pode alterar a intensidade do treinamento. Apesar de não haver estudos relacionando diretamente a velocidade de execução com o EPOC, como há relação com a intensidade, é provável que haja influência no EPOC.

O número de séries de um exercício de resistência varia conforme o objetivo pretendido, porém, há recomendações relacionadas com a saúde, como da American Heart Association (2001) para a realização de pelo menos uma série de 8 a 15 repetições para os principais grupos musculares. Estudos que se baseiam em aspectos relacionados à saúde demonstram que programas de série única podem levar a ganhos de força e hipertrofia muscular de forma similar a series múltiplas, principalmente em indivíduos sedentários ou na fase inicial de um programa de treinamento (WOLFE et al., 2004).

Quanto à influência do número de séries no EPOC, estudos de Henley et al. (2004) e Haddock e Wilkins (2006) demonstraram que o gasto energético total de três séries foi, aproximadamente, três vezes maior que o gasto energético de uma série. Entretanto, o gasto foi semelhante quando os valores foram expressos em relação ao tempo de exercício. Já o EPOC foi de igual magnitude em valores absolutos e maior na situação de série única, quando expresso por tempo de exercício. 
Como pode ser observado, o gasto energético absoluto aumenta de acordo com o número de séries realizadas, no entanto, o gasto parece não ter diferença quando se considera o tempo de duração do estimulo. Já, quando se considera a magnitude do EPOC, o consumo parece não ser muito afetado pelo número de séries.

\section{Exercício em cicloergômetro e EPOC}

Existem poucos estudos realizados em cicloergômetros com o objetivo de medir o EPOC. Em um deles, Lyons et al. (2007) avaliaram exercício realizado em cicloergômetros na intensidade de $60 \%$ do consumo máximo de oxigênio $\left(\mathrm{VO}_{2}\right.$ máx. $)$ durante $20 \mathrm{~min}$, tendo observado que o consumo de oxigênio após o exercício foi de 2,9 L/min, em média, num período de 16 minutos até o retorno ao consumo de repouso.

\section{CONSIDERAÇÕES FINAIS}

Mesmo com a grande dificuldade de medida do consumo de oxigênio durante as atividades realizadas na água, há alguns estudos sobre $O$ EPOC relatando sua magnitude $e$ duração, além da importância de contribuir com uma proporção do gasto energético total.

Em exercícios resistidos, há mais variáveis que podem influenciar a magnitude e duração do EPOC, entre elas o tipo de exercício realizado e a massa muscular envolvida, o número de séries e repetições, o intervalo entre as séries e a intensidade e duração da sessão de treinamento. Porém, a intensidade é considerada o fator que mais influência o EPOC em exercícios de resistência.

Nos exercícios aquáticos, em geral, há sempre o trabalho de massa muscular mais ampla, seja para realização dos exercícios ou para manutenção do equilíbrio, visto que a água é um meio mais instável. Em razão disto, os exercícios aquáticos, como hidroginástica e, principalmente, natação podem apresentar EPOC muito superior comparativamente aos exercícios de resistência e em cicloergômetro.

Assim, pode-se concluir que o EPOC sofre influência de vários fatores, principalmente do tipo de exercício, intensidade e duração. Esses dois últimos podem fazer com que o EPOC represente um consumo adicional de oxigênio correspondente a $10-20 \%$ daquele que ocorreu durante a sessão de exercício.

\section{CONFLITO DE INTERESSE}

Os autores declaram não haver qualquer potencial conflito de interesse que possa interferir na imparcialidade deste trabalho científico.

\section{REFERÊNCIAS}

Alves RV, Mota J, Costa MC, Alves JGB. Physical fitness and elderly health effects of hydrogymnastics. Rev Bras Med Esporte. 2004;10:38-43.

American Heart Association. Exercise standards for healthcare professionals from the American Heart Association. Circulation. 2001;104(14):1694-740. http://dx.doi.org/10.1161/hc3901.095960

Barella RE, Muller FG, Severo CR, Cardoso AS, Figueiredo PAP, Brentano MA, et al. Efeitos de um treinamento de força aplicado em mulheres praticantes de hidroginástica. Rev Bras Fisiol Exerc. 2004;3:136.

Baum G. Aquaeróbica: manual de treinamento. Ed. Manole, 2000.

Bøsheimm E, Knardahl S, Hostmark AT, Bahr R. Adrenergic control of post-exercise metabolism. Acta Physiol Scand. 1998;162(3):313-23. http://dx.doi.org/10.1046/j.1365201X.1998.0321e.x

Dolezal BA, Potteing JA, Jacobse DJ, Benedict $\mathrm{SH}$. Muscle damage and resting metabolic rate after acute resistance exercise with an eccentric overload. Med Sci Sports Exerc. 2000;32(7):12027. http://dx.doi.org/10.1097/00005768-200007000$\underline{00003}$

Foureaux G, Pinto KMC, Damaso A. Efeito do consumo excessivo de oxigênio após exercício e da taxa metabólica de repouso no gasto 
energético. Rev. Bras Med Esporte. 2006;12(6):393-8.

http://dx.doi.org/10.1590/S1517$\underline{86922006000600018}$

Gaesser GA, Brooks GA. Metabolic bases of excess post-exercise oxygen consumption: a review. Med Sci Sports Exerc. 1984;16(1):29-43. http://dx.doi.org/10.1249/00005768-198401000$\underline{00008}$

Haddock BI, Wilkins LD. Resistance training volume and postexercise energy expenditure. Int J Sports Med. 2006;27:143-8. http://dx.doi.org/10.1055/s-2005-865601

Haltom RW, Kraemer RR, Sloan RA, Hebert EP, Frank K, Tryniecki JL. Circuit weight training and its effects on excess postexercise oxygen consumption. Med Sci Sports Exerc. 1999;31(11):1613-8.

http://dx.doi.org/10.1097/00005768-199911000$\underline{00018}$

Henley MO, Irving BA, Gaesser GA. Effect of single and multiple-set resistance exercise on postexercise energy expenditure [abstract]. Med Sci Sports Exerc. 2004;36(5):5277.

Imamura H, Shibuya S, Uchida K, Teshima K, Masuda R, Miyamoto N. Effect of moderate exercise on excess post-exercise oxygen consumption and catecholamines in young women. J Sports Med Phys Fitness. 2004;44:239.

Kenny GP, Reardson FD, Zaleski W, Reardson ML, Haman F, Ducharme MB, Muscle temperature transients before, during, and after exercise using an intramuscular multisensor probe. J Appl Physiol. 2003;94(6):2350-7.

Keskinen KL, Rodríguez FA, Keskinen OP. Respiratory snorkel and valve system for breathby-breath gas analysis in swimming. Scand J Med Sci Sports. 2003;13:322-9.

http://dx.doi.org/10.1034/i.1600-

$\underline{0838.2003 .00319 . x}$

Kruel LFM. Alterações fisiológicas e biomecânicas em indivíduos praticando exercícios de hidroginástica dentro e fora d'água. Santa Maria, 2000. Tese de Doutorado. Universidade Federal de Santa Maria.

LeCheminant JD, Jacobsen DJ, Bailey BW, Mayo MS, Hill JO, Smith BK, Donnelly JE. Effects of longterm aerobic exercise on EPOC. Int J Sports Med. 2008;29(1):53-8. http://dx.doi.org/10.1055/s2007-965111

Lyons S, Richardson M, Bishop P, Smith J, Heath $\mathrm{H}$, Giesen J. Excess post-exercise oxygen consumptions in untrained men following exercise of equal energy expenditure: comparisons of upper and lower body exercise. Diabetes Obes Metab. http://dx.doi.org/10.1111/j.14631326.2006.00679.x

Matsuura C, Meirelles CM, Gomes PSC. Gasto energético e consumo de oxigênio pós-exercício contra-resistência. Rev Nutr. 2006;19(6):729-40. http://dx.doi.org/10.1590/S1415$\underline{52732006000600009}$

McArdle W, Katch F, Katch V. Fisiologia do Exercício. 6ed. Rio de Janeiro: GuanabaraKoogan; 2008.

McComb JR, Daesung $\mathrm{R}$, Williams $\mathrm{J}$. The prediction of maximal oxygen consumption from a submaximal water running test. Med Sci Sports Exerc. 2004;36:S90.

Meirelles CM, Gomes PSC. Efeitos agudos da atividade contra-resistência sobre 0 gasto energético: revisando o impacto das principais variáveis. Rev Bras Med Esporte. 2004;10(2):12230.

Miyoshi T, Shirota T, Yamamoto S, Nakazawa K, Akai M. Effect of the walking speed to the lower limb joint angular displacements, joint moments and ground reaction forces during walking in water. Disabil Rehabil. 2004;26:724-32. http://dx.doi.org/10.1080/09638280410001704313

Muller FG. A treinabilidade da força muscular em idosas praticantes de hidroginástica. Florianópolis, 2002. Dissertação de Mestrado. Universidade do Estado de Santa Catarina.

Pinto SS, Alberton CL, Becker ME, Olkoski MM, Kruel LFM. Respostas cardiorrespiratórias em exercícios de hidroginástica executados com e sem o uso de equipamento resistivo. Rev Port Cienc Desp. 2006;6:336-41.

Schuenke MD, Mikat P, McBride JM. Effect of an acute period of resistance exercise on excess post-exercise oxygen consumption: implications for body mass management following a bout of heavy resistance exercise. Eur J Appl Physiol. 2002;86(5):411-7.

http://dx.doi.org/10.1007/s00421-001-0568-y

Takeshima N, Rogers ME, Watanabe WF, Brechue WF, Okada A, Yamada T, et al. Waterbased exercise improves health-related aspects of fitness in older women. Med Sci Sports Exerc. 2002;33:544-51.

http://dx.doi.org/10.1097/00005768-200203000$\underline{00024}$ 
Thorton MK, Potteiger JA. Effects of resistance exercise bouts of different intensities but equal work on EPOC. Med Sci Sports Exerc. 2002;34(4):715-22.

http://dx.doi.org/10.1097/00005768-200204000$\underline{00024}$

Toussaint HM, Meulemans A, Groot G, Hollander AP, Schreurs AW, Vervoorn K. Respiratory valve for oxygen uptake measurements during swimming. Eur J Appl Physiol. 1987;56:363-6. http://dx.doi.org/10.1007/BF00690906

Wolfe BL, Lemura LM, Cole PJ. Quantitative analysis of single vs. multiple set programs in resistance training. J Strenght Cond Res. 2004;18(1):35-47. 